\title{
Treating to the target of remission in early rheumatoid arthritis is cost-effective: results of the DREAM registry
}

\author{
Marloes Vermeer ${ }^{1,2}$, Wietske Kievit ${ }^{3}$, Hillechiena H Kuper ${ }^{2 *}$, Louise MA Braakman-Jansen ${ }^{1}$, Hein J Bernelot Moens ${ }^{4}$,
} Theo R Zijlstra ${ }^{5}$, Alfons A den Broeder ${ }^{6}$, Piet LCM van Riel ${ }^{3}$, Jaap Fransen ${ }^{3}$ and Mart AFJ van de Laar ${ }^{1,2}$

\begin{abstract}
Background: Where health economic studies are frequently performed using modelling, with input from randomized controlled trials and best guesses, we used real-life data to analyse the cost-effectiveness and costutility of a treatment strategy aiming to the target of remission compared to usual care in early rheumatoid arthritis (RA).

Methods: We used real-life data from comparable cohorts in the Dutch Rheumatoid Arthritis Monitoring (DREAM) registry: the DREAM remission induction cohort (treat-to-target, T2T) and the Nijmegen early RA inception cohort (usual care, UC). Both cohorts were followed prospectively using the DREAM registry methodology. All patients fulfilled the American College of Rheumatology criteria for RA and were included in the cohort at the time of diagnosis. The T2T cohort was treated according to a protocolised strategy aiming at remission (Disease Activity Score in 28 joints (DAS28) < 2.6). The UC cohort was treated without DAS28-guided treatment decisions. EuroQol-5D utility scores were estimated from the Health Assessment Questionnaire. A health care perspective was adopted and direct medical costs were collected. The incremental cost effectiveness ratio (ICER) per patient in remission and incremental cost utility ratio (ICUR) per quality-adjusted life year (QALY) gained were calculated over two and three years of follow-up.
\end{abstract}

Results: Two year data were available for 261 T2T patients and 213 UC patients; an extended follow-up of three years was available for 127 and 180 patients, respectively. T2T produced higher remission percentages and a larger gain in QALYs than UC. The ICER was $€ 3,591$ per patient in remission after two years and T2T was dominant after three years. The ICUR was $€ 19,410$ per QALY after two years and T2T was dominant after three years.

Conclusions: We can conclude that treating to the target of remission in early RA is cost-effective compared with UC. The data suggest that in the third year, T2T becomes cost-saving.

\section{Background}

Treating to the target of remission has become the new paradigm for the treatment of patients with rheumatoid arthritis (RA) [1]. The key elements of treat-to-target (T2T) are: monitoring disease activity, subsequently adjusting medication in accordance to a fixed protocol, and aiming at a predefined target. In clinical trials it has been demonstrated that a T2T approach is more effective

\footnotetext{
*Correspondence: h.kuper@mst.nl

${ }^{2}$ Department of Rheumatology and Clinical Immunology, Medisch Spectrum Twente, Enschede, The Netherlands

Full list of author information is available at the end of the article
}

in lowering disease activity and, ultimately, reaching remission than usual care [2-7].

Taking into account that treating RA comes with potential high costs, it is mandatory to study the balance between costs and effects and ultimately gained quality of life. Health economic studies addressing this question frequently use modelling as methodology to evaluate this balance. These studies use data from pivotal trials and best guesses by opinion leaders to feed the model. For prediction before or early after introducing innovations to the market, modelling is a realistic approach. However, clinical trial data, clinical experience and mathematical models have their restrictions. Therefore, real-life

\section{() Biomed Central}


data are needed to study the economic impact of innovations in health care compared with usual care.

In the Dutch Rheumatoid Arthritis Monitoring (DREAM) registry, 11 centres prospectively acquire standardized data on their RA patients. In the DREAM registry, centres participate in different levels and cohorts. One of the DREAM cohorts is the DREAM remission induction cohort. With this cohort we have demonstrated that a T2T strategy aiming for remission (Disease Activity Score in 28 joints (DAS28) $<2.6$ [8]) is very effective in daily clinical practice, with percentages of DAS28 remission ranging from $47 \%$ after six months to $58 \%$ after twelve months [9]. In this early RA cohort, remission was achieved rapidly with a median time to first remission of 25 weeks. Moreover, this T2T strategy resulted in beneficial clinical outcomes after one year compared to usual care treatment [7].

Early and effective suppression of disease activity is expected to reduce pain, prevent progression of joint damage and disability $[10,11]$, and increase the patient's quality of life $[12,13]$. The concept of T2T assumes that intensive efforts and costs are made in the beginning of the disease to gain health and cost savings later. However, the question is whether indeed the health benefits outweigh the extra costs associated with performing a T2T approach.

The objective of this health economic study is to evaluate the cost-effectiveness and cost-utility, from a health care perspective, of a T2T strategy aiming at remission compared to usual care for the treatment of early RA patients in real-life daily clinical practice over a period of up to three years.

\section{Methods}

\section{Study design}

The data in this study are prospectively acquired in participating centres of the DREAM registry. Post-hoc we analysed the data of two cohorts. All DREAM centres are stationed in the eastern part of The Netherlands and have the same health care and reimbursement system. The T2T cohort consisted of patients from the DREAM remission induction cohort and the usual care (UC) cohort consisted of patients from the Nijmegen early RA inception cohort [14]. This study can be defined as a quasi-experiment because unselected patients were included in both cohorts with 'living area' as main determinant for being included in either one of the cohorts. In both cohorts, all clinical data on patient characteristics, medication, clinical and laboratory measures were assessed in a standardized way and stored prospectively in electronic databases. Currently, in both cohorts, new patients are still being included and follow-up continues. The local medical ethics committees have approved the prospective data acquisition of both inception cohorts (Medisch Spectrum Twente Hospital for the T2T cohort and CMO region Arnhem-Nijmegen for the usual care cohort). Each patient gave informed consent before the inclusion in the cohorts.

\section{DREAM registry - treat-to-target}

Since January 2006, patients were enrolled in the DREAM remission induction cohort [9]. A T2T strategy including standardised and protocolised treatment adjustments aiming at remission (DAS28 $<2.6)$ was applied. Patients visited the clinic at weeks $0,8,12,20,24,36$ and 52, and every three months thereafter. Therapy consisted of initial methotrexate monotherapy (MTX), followed by the addition of sulfasalazine (SSZ), and thereafter in the case of persistent disease activity, sulfasalazine was replaced with anti-tumour necrosis factor (TNF) $\alpha$ agents. If the target of DAS $28<2.6$ was met, medication was not changed. In case of sustained remission $(\geq$ six months), medication was gradually reduced and eventually discontinued. Nonsteroidal anti-inflammatory drugs (NSAIDs), prednisolone at a dosage of $\leq 10 \mathrm{mg} /$ day, and intra-articular corticosteroid injections were allowed at the discretion of the attending rheumatologist. Data collection, including assessment of the DAS28, was performed by trained rheumatology nurses.

\section{DREAM registry - usual care}

Follow-up and data acquisition for the usual care cohort were similar to the T2T cohort in the DREAM registry. Every three months, the DAS28 was assessed by trained rheumatology nurses. In contrast to a T2T approach, the DAS28 values were not generally provided to the treating rheumatologist and pharmacological treatment was not protocolised but at the discretion of the rheumatologist. In general, patients were treated with step-up or sequential monotherapy with conventional disease-modifying antirheumatic drugs (DMARDs) and/or biologic, notably antiTNF. Prednisolone (oral or injections) and NSAIDs could also be used. The most commonly applied strategy was starting with MTX monotherapy, subsequently switching to SSZ or adding SSZ in case of MTX failure, and adding an anti-TNF agent after two or more DMARDs had failed.

\section{Selection of patients}

For the current study, we selected patients from both cohorts who fulfilled the following inclusion criterion: RA according to the American College of Rheumatology 1987 classification criteria for RA [15], age $\geq 18$ years, symptom duration of less than one year, and no previous treatment with DMARDs. Patients diagnosed between January 2000 and February 2009 with a minimal followup of two years were selected. The DREAM remission induction cohort started in 2006. Although we hoped to have sufficient data on UC in a comparable time slot, we had to include patients for the UC group diagnosed 
from January 2000 onwards in order to obtain a sufficient number of patients. We chose 2000 as a minimum year of inclusion because since then biologic agents, especially TNF blockers, were already available and reimbursed with restriction in daily practice.

Patients from the T2T cohort visited Arthritis Center Twente at Medisch Spectrum Twente, Enschede and the departments of rheumatology from Ziekenhuisgroep Twente, Almelo/Hengelo and Isala Klinieken, Zwolle. Patients from the UC cohort visited the Radboud University Nijmegen Medical Centre or Maartenskliniek, Nijmegen.

\section{Measurements}

The effectiveness of treatment was evaluated using the DAS28 (calculated using the erythrocyte sedimentation rate (ESR)). A DAS28 < 2.6 was defined as remission [16].

Utilities were estimated to evaluate the effect of treatment on health-related quality of life. Utility is the valuation of a health state on a scale of 0 (equivalent to death) to 1 (equivalent to full health) and is used to derive quality-adjusted life years (QALYs) [17]. Because preference based measures were not prospectively assessed, EuroQol-5D (EQ-5D) values [18] were estimated from the Health Assessment Questionnaire (HAQ) scores [19,20] by using model 5 of the mapping method by Bansback et al. [21]. This model was reported to be the most successful of the five mapping methods, by having the lowest mean square error and the best predictive value $[21,22]$. Concordantly, the QALYs were computed according to the trapezium rule.

\section{Cost analysis}

The cost analysis exists of two main parts. First, on patient level, volumes of care related to the T2T strategy or usual care were determined. Volumes of hospital related care, i.e. consultations with the rheumatologist and the rheumatology nurse, telephonic consultations (rheumatologist), and hospital admissions related to RA, were retrieved from the hospital information system. Medication use (exact dose of medication and administration period) was prospectively registered in the electronic case report forms.

The second part of the cost analysis consisted of determining the cost prices for each volume of consumption. Volumes of care were multiplied by the cost prices for each volume of care to calculate costs. The standard cost prices from the Dutch Guideline for Cost Analyses were used for hospital related care (see Appendix) [23]. The price based on personnel, material and overhead of day care hospital admissions required for treatment with infliximab or rituximab was estimated at a mean of $€$ 122 per day (on top of the medication costs). Cost prices for medication were retrieved from the Dutch national tariff list provided by the Dutch Board of Health Insurances [23].
The base year was 2011 for all prices. Prices retrieved from other years were converted to 2011 euros using the general Dutch price index rate [24].

\section{Statistical analysis}

Data of two year follow-up were analysed as well as an extended follow-up of three years in patients who had sufficient follow-up. Our expectation was that on the long-term, costs associated with performing T2T will decrease. In our previous study, the necessary sample size to detect a difference in remission of $20 \%$ between both groups was estimated to be at least $2 \times 125=250$ patients [7]. This sample size estimation was satisfied in the two and three years data analyses in the present study.

The incremental cost-effectiveness ratio (ICER) was calculated by dividing the difference in costs by the difference in effectiveness (based on the number of patients in remission) derived from the two groups. The ICER is expressed as costs per one more patient in remission. The incremental cost-utility ratio (ICUR) was calculated by dividing the difference in costs by the difference in the QALYs produced by the two groups. The ICUR is expressed as costs per QALY gained. Uncertainty in both ratios was determined non-parametrically using bootstrap techniques. Results of the 1,000 bootstrapped replications are presented in cost-effectiveness planes that graphically present the uncertainty around the ratio of the two and three years data. In a sub-analysis, the ratios were calculated for patients that were included in the cohorts from 2006. However, statistical power was expected to be low due to the low number of UC patients.

Missing values were imputed with single imputation using a regression method including a random component for the ESR, patient's assessment of general health, and HAQ question 23 (take a tub bath) or linear interpolation using the trapezoid method for the DAS28 and EQ-5D scores, conditional on the data being missing at random.

The level of significance was set at a $\mathrm{p}$ value $<0.05$. Statistical analyses were performed using the statistical software package SPSS 18.0 (SPSS Inc., Chicago, IL, USA). The bootstrap was performed in Excel.

\section{Results}

\section{Baseline characteristics}

Two year follow-up data were available for 261 patients of the T2T cohort and for 213 patients of the UC cohort. An extended three years follow-up was available for a smaller proportion of patients due to insufficient followup; i.e. in 127 of the 261 (48.7\%) T2T patients and 180 of the 213 (84.5\%) UC patients. Baseline characteristics were comparable between patients with and without sufficient follow-up.

Table 1 presents the demographic and clinical characteristics of both groups at baseline. The groups were 
Table 1 Baseline characteristics of the patients of the treat-to-target (T2T) and usual care (UC) groups

\begin{tabular}{lccc}
\hline & T2T $(\mathbf{n}=\mathbf{2 6 1})$ & UC (n=213) & UC from 2006 (n=69) \\
\hline Age, mean \pm SD years & $57.9 \pm 13.8$ & $56.6 \pm 13.4$ & $53.9 \pm 13.0+$ \\
Female sex, $n(\%)$ & $161(61.7)$ & $132(62.0)$ & $43(62.3)$ \\
RF positive, $n(\%)$ & $178(68.2)$ & $147 / 211(69.7)$ & $48 / 69(69.6)$ \\
DAS28, mean \pm SD & $5.0 \pm 1.1$ & $4.8 \pm 1.2+$ & $4.8 \pm 1.3$ \\
No. of swollen joints (28 assessed), median (IQR) & $8(5-12)$ & $9(6-13) \dagger$ & $8(5-12)$ \\
No. of tender joints (28 assessed), median (IQR) & $5(2-9)$ & $4(2-9)$ & $4(1-9)$ \\
ESR, median (IQR) mm/hour & $28.0(15.5-42.0)$ & $26.0(12.0-39.0)$ & $29.0(17.0-43.5)$ \\
CRP, median (IQR) mg/litre & $14.0(5.0-34.5)$ & $6.7(0.0-27.8) \dagger$ & $10.0(0.0-34.3)$ \\
VAS general health, mean \pm SD (0-100) & $52.9 \pm 22.6$ & $45.7 \pm 23.0 \dagger$ & $42.9 \pm 24.4 \dagger$ \\
VAS pain, mean \pm SD (0-100) & $51.2 \pm 21.9$ & $44.9 \pm 23.2 \dagger$ & $46.5 \pm 23.9$ \\
HAQ score, median (IQR) & $1.1(0.6-1.5)(n=244)$ & $0.9(0.5-1.4)(n=151) \dagger$ & $0.9(0.4-1.4)(n=52) \dagger$ \\
\hline
\end{tabular}

CRP, C-reactive protein; DAS28, Disease Activity Score in 28 joints; ESR, erythrocyte sedimentation rate; HAQ, Health Assessment Questionnaire; IQR, interquartile range; $\mathrm{RF}$, rheumatoid factor; $\mathrm{SD}$, standard deviation; VAS, visual analog scale.

$+\mathrm{P}<0.05$ for differences between groups (T2T versus UC).

comparable at baseline regarding age, gender, rheumatoid factor (RF) positivity, number of tender joints (28 assessed) and ESR. Statistically significant but small differences were found for the mean DAS28, number of swollen joints (28 assessed), C-reactive protein, patient's assessment of general health and pain, and HAQ score, which were higher in the T2T group.

MTX monotherapy was the initial treatment in the T2T group by protocol. In the UC group, patients started with SSZ monotherapy $(45.5 \%, 97 / 213)$, MTX monotherapy $(43.7 \%, 93 / 213)$ or occasionally another DMARD $(6.6 \%, 14 / 213)$ or no medication $(4.2 \%, 9 / 213)$.

\section{Health outcomes}

Table 2 presents the health outcome results after two and three years of follow-up. After two years, $64.4 \%$ $(168 / 261)$ of the T2T group was in remission versus
$34.7 \%(74 / 213)$ of the UC group $(\mathrm{p}<0.001)$. Over the first two years of treatment, the median (interquartile range, IQR) of QALYs was higher in the T2T group than in the UC group (1.45 (1.24-1.55) versus 1.39 (1.181.53), respectively, $\mathrm{p}=0.04$ ).

After three years, the remission percentages were 59.8\% (76/127) with T2T versus 35.0\% (63/180) with UC ( $\mathrm{p}<0.001)$. The median (IQR) of QALYs over the first three years was higher in the T2T group than in the UC group (2.19 (1.81-2.34) versus $2.04(1.64-2.27)$, respectively, $\mathrm{p}=0.05)$.

\section{Care consumption and costs}

Table 3 presents the amount of care consumption and mean costs per patient during two and three years of follow-up. Over both periods, the numbers of consultations with the rheumatologist were comparable, whereas

Table 2 Health outcomes in the treat-to-target (T2T) and usual care (UC) groups after two and three years of follow-up

\begin{tabular}{|c|c|c|c|c|c|c|}
\hline & \multicolumn{3}{|c|}{ Two years } & \multicolumn{3}{|c|}{ Three years } \\
\hline & T2T $(n=261)$ & $U C(n=213)$ & $\begin{array}{c}\text { UC from } 2006 \\
(n=69)\end{array}$ & T2T $(n=127)$ & UC $(n=180)$ & $\begin{array}{c}\text { UC from } 2006 \\
(n=45)\end{array}$ \\
\hline DAS28, mean $\pm S D$ & $2.4 \pm 1.0$ & $3.1 \pm 1.3+$ & $2.8 \pm 1.1 \dagger$ & $2.5 \pm 1.0$ & $3.1 \pm 1.3+$ & $2.7 \pm 1.1$ \\
\hline \multicolumn{7}{|l|}{ DAS28 level, n (\%) } \\
\hline $\begin{array}{l}\text { Remission } \\
(\text { DAS28 < 2.6) }\end{array}$ & $168(64.4)$ & $74(34.7) \dagger$ & $33(47.8) \dagger$ & $76(59.8)$ & $63(35.0) \dagger$ & $25(55.6)$ \\
\hline $\begin{array}{l}\text { Low } \\
(2.6 \leq \mathrm{DAS} 28 \leq 3.2)\end{array}$ & $48(18.4)$ & $44(20.7)$ & $14(20.3)$ & $28(22.0)$ & $35(19.4)$ & $5(11.1)$ \\
\hline $\begin{array}{l}\text { Moderate } \\
(3.2<\text { DAS28 } \leq 5.1)\end{array}$ & $37(14.2)$ & $76(35.7) \dagger$ & $19(27.5) \dagger$ & $21(16.5)$ & $72(40.0)+$ & $14(31.1) \dagger$ \\
\hline High (DAS28 > 5.1) & $8(3.1)$ & $19(8.9) \dagger$ & $3(4.3)$ & $2(1.6)$ & $10(5.6)$ & $1(2.2)$ \\
\hline QALYS, median (IQR) & $\begin{array}{c}1.45(1.24-1.55) \\
(n=221)\end{array}$ & $\begin{array}{c}1.39 \begin{array}{c}(1.18-1.53) \\
(n=143) \dagger\end{array}\end{array}$ & $\begin{array}{c}1.44 \begin{array}{c}(1.21-1.55) \\
(n=47)\end{array}\end{array}$ & $\begin{array}{c}2.19(1.81-2.34) \\
\quad(n=101)\end{array}$ & $\begin{array}{c}2.04(1.64-2.27) \\
(n=106) \dagger\end{array}$ & 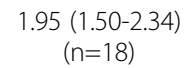 \\
\hline
\end{tabular}

Quality-adjusted life years (QALYs) were derived from the EuroQol-5D utility scores which were estimated from the Health Assessment Questionnaire (HAQ).

QALYs could not be evaluated in all patients due to missing data in (items of) the HAQ.

DAS28, Disease Activity Score in 28 joints; SD, standard deviation.

† $\mathrm{P}<0.05$ for differences between groups (T2T versus UC). 
Table 3 Mean volumes of care and total direct costs in euros per patient per period in the treat-to-target (T2T) and usual care (UC) groups after two and three years of follow-up

\begin{tabular}{|c|c|c|c|c|c|c|c|c|}
\hline & \multicolumn{8}{|c|}{$0-2$ year } \\
\hline & \multicolumn{2}{|c|}{ T2T $(n=261)$} & \multicolumn{2}{|c|}{$U C(n=213)$} & \multirow[b]{2}{*}{$\begin{array}{l}\text { Difference } \\
\text { in costs }\end{array}$} & \multicolumn{2}{|c|}{ UC from $2006(n=69)$} & \multirow[b]{2}{*}{$\begin{array}{l}\text { Difference } \\
\text { in costs }\end{array}$} \\
\hline & Volume & Costs & Volume & Costs & & Volume & Costs & \\
\hline $\begin{array}{l}\text { Consultations } \\
\text { rheumatologist }\end{array}$ & $10.4 \pm 3.0$ & $696 \pm 199$ & $10.3 \pm 2.9$ & $689 \pm 195$ & 7 & $10.2 \pm 3.6$ & $683 \pm 239$ & 13 \\
\hline $\begin{array}{l}\text { Consultations } \\
\text { nurse }\end{array}$ & $8.8 \pm 3.0$ & $588 \pm 200$ & $7.8 \pm 1.9 \dagger$ & $522 \pm 127 \dagger$ & 66 & $6.6 \pm 2.0+$ & $438 \pm 134 \dagger$ & 150 \\
\hline $\begin{array}{l}\text { Telephonic } \\
\text { consultations }\end{array}$ & $1.3 \pm 1.8$ & $34 \pm 46$ & $0.6 \pm 1.4 \dagger$ & $16 \pm 36 t$ & 18 & $1.1 \pm 1.9$ & $29 \pm 48$ & 5 \\
\hline $\begin{array}{l}\text { Hospital } \\
\text { admissions }\end{array}$ & $0.4 \pm 2.7$ & $178 \pm 1,208$ & $1.1 \pm 4.2 \dagger$ & $521 \pm 1,901 \dagger$ & -343 & $0.3 \pm 1.6$ & $158 \pm 714$ & 20 \\
\hline \multicolumn{9}{|l|}{ Medication } \\
\hline DMARDs/other & & $174 \pm 165$ & & $249 \pm 340$ & -75 & & $166 \pm 119$ & 8 \\
\hline Anti-TNF & & $3,121 \pm 7,162$ & & $1,730 \pm 4,905 \dagger$ & 1,391 & & $1,877 \pm 5,086$ & 1244 \\
\hline \multirow[t]{4}{*}{ Total } & & $4,791 \pm 7,436$ & & $3,727 \pm 5,773$ & $\begin{array}{c}1,064 \\
(1,026 \text { to } 1,121) \neq\end{array}$ & & $3,351 \pm 5,179+$ & $\begin{array}{c}1440 \\
(1,387 \text { to } 1479) \neq\end{array}$ \\
\hline & \multicolumn{8}{|c|}{$0-3$ year } \\
\hline & \multicolumn{2}{|c|}{ T2T $(n=127)$} & \multicolumn{2}{|c|}{$U C(n=180)$} & & \multicolumn{2}{|c|}{ UC from $2006(n=45)$} & \\
\hline & Volume & Costs & Volume & Costs & $\begin{array}{l}\text { Difference } \\
\text { in costs }\end{array}$ & Volume & Costs & $\begin{array}{l}\text { Difference } \\
\text { in costs }\end{array}$ \\
\hline $\begin{array}{l}\text { Consultations } \\
\text { rheumatologist }\end{array}$ & $13.7 \pm 3.3$ & $917 \pm 220$ & $13.6 \pm 3.3$ & $909 \pm 224$ & 8 & $12.5 \pm 3.8$ & $838 \pm 253+$ & 79 \\
\hline $\begin{array}{l}\text { Consultations } \\
\text { nurse }\end{array}$ & $12.1 \pm 3.6$ & $809 \pm 244$ & $8.9 \pm 1.7 \dagger$ & $596 \pm 117 \dagger$ & 213 & $8.4 \pm 2.2 \dagger$ & $565 \pm 145 \dagger$ & 244 \\
\hline $\begin{array}{l}\text { Telephonic } \\
\text { consultations }\end{array}$ & $1.9 \pm 2.1$ & $49 \pm 54$ & $0.8 \pm 1.7 \dagger$ & $21 \pm 45 \dagger$ & 28 & $1.7 \pm 2.5$ & $43 \pm 64$ & 6 \\
\hline $\begin{array}{l}\text { Hospital } \\
\text { admissions }\end{array}$ & $0.5 \pm 3.2$ & $215 \pm 1,441$ & $1.6 \pm 5.0 \dagger$ & $748 \pm 2,263 \dagger$ & -533 & $0.8 \pm 2.7$ & $364 \pm 1,217$ & -149 \\
\hline \multicolumn{9}{|l|}{ Medication } \\
\hline DMARDs/other & & $260 \pm 335$ & & $423 \pm 612+$ & -163 & & $259 \pm 174$ & 1 \\
\hline Anti-TNF & & $4,160 \pm 10,685$ & & $4,175 \pm 10,070$ & -15 & & $5,488 \pm 11,528$ & $-1,328$ \\
\hline Total & & $6,410 \pm 10,845$ & & $6,872 \pm 11,033$ & $\begin{array}{c}-462 \\
(-513 \text { to }-350) \neq\end{array}$ & & $7,558 \pm 11,649$ & $\begin{array}{c}-1,148 \\
(-1241 \text { to }-1075) \neq\end{array}$ \\
\hline
\end{tabular}

Volumes are the mean \pm standard deviation (SD).

Anti-TNF, anti-tumour necrosis factor; $\mathrm{Cl}$, confidence interval; DMARDs, disease-modifying antirheumatic drugs.

$+\mathrm{P}<0.05$ for differences between groups (T2T versus UC). $\neq 95 \%$ confidence interval bootstrapped.

the numbers of consultations with the rheumatology nurse and telephonic consultations were higher in the $\mathrm{T} 2 \mathrm{~T}$ group. In usual care, more hospital admissions were observed than with $\mathrm{T} 2 \mathrm{~T}$.

Over the first two years, the mean (standard deviation, SD) total direct costs per patient were $€ 4,791(7,436)$ in the T2T group and $€ 3,727(5,773)$ in the usual care group (Table 3). The observed difference in costs between groups was mainly generated by the costs of antiTNF therapy and hospitalization. During the first two years of treatment, $21.5 \%(56 / 261)$ of the T2T group received anti-TNF therapy versus $15.0 \%(32 / 213)$ of the $\mathrm{UC}$ group.
Over the first three years, the mean (SD) total direct costs per patient were $€ 6,410(10,845)$ in the T2T group and $€ 6,872(11,033)$ in the UC group (Table 3$)$. The observed difference in costs between groups was mainly generated by hospitalization.

Overall, the mean (SD) time until the first anti-TNF agent was started was shorter in the T2T group compared to the UC group (mean (SD) of 58 (29) weeks versus 80 (39) weeks, respectively, $\mathrm{p}=0.002$ ).

\section{Cost-effectiveness}

After two years of follow-up, the ICER was $€ 3,591$ per patient in remission and after three years of follow-up 


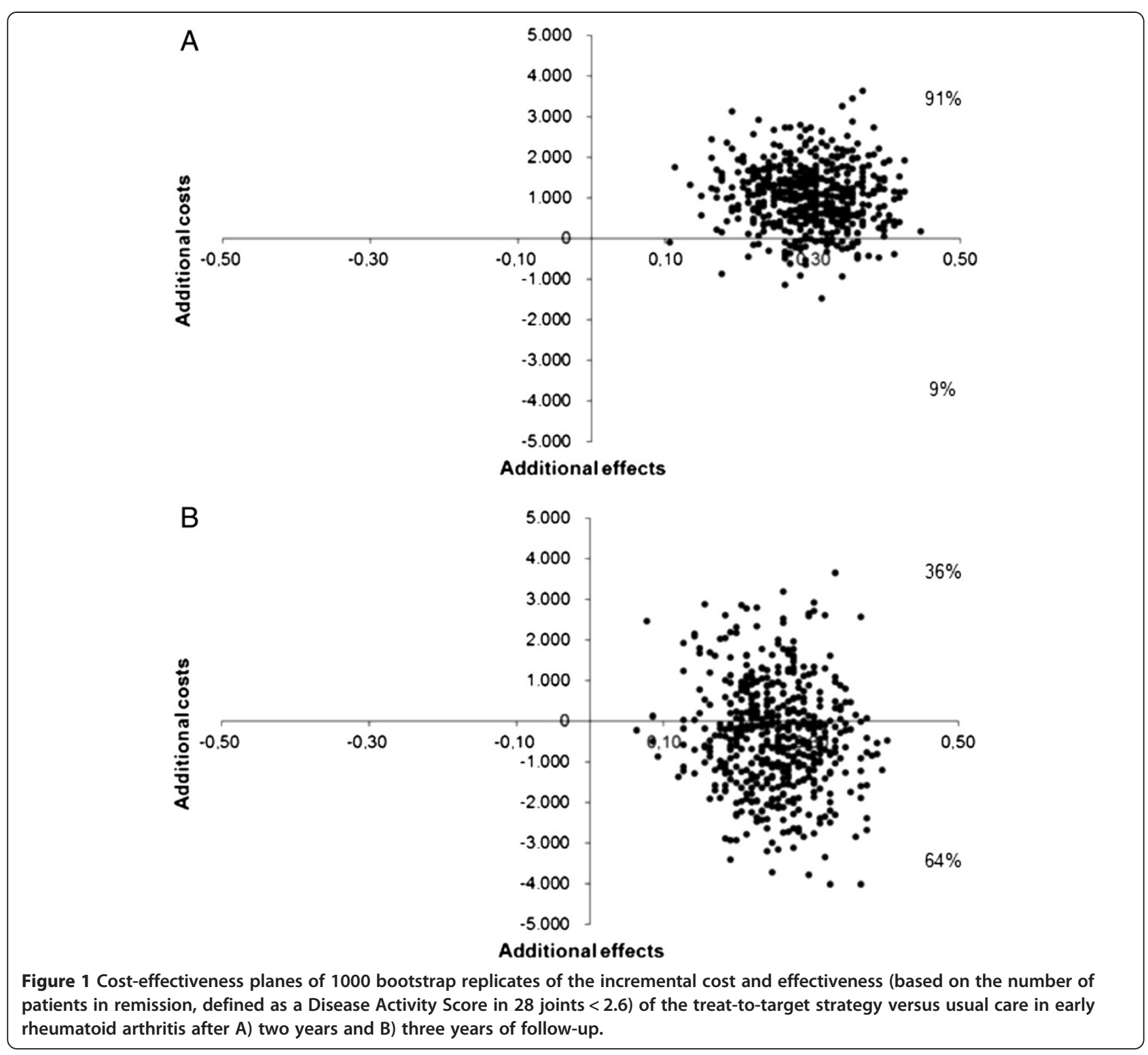

the T2T strategy was dominant. Figure 1 presents the cost-effectiveness planes, showing the relation between the difference in effect ( $x$-axis) and the difference in costs (y-axis) between T2T and UC. Figure 1A presents the two years data and shows that $91 \%$ of the bootstrapped ratios were situated in the upper-right quadrant, which signifies a gain in effectiveness against higher costs. Figure 1B shows that after three years, $64 \%$ of the bootstrapped ratios were situated in the lower-right quadrant, which signifies lower costs and higher effectiveness.

\section{Cost-utility}

Over a period of two years, the ICUR was $€ 19,410$ per QALY and after three years of treatment the T2T strategy was dominant. Figure 2 presents the cost-utility planes, showing the relation between the difference in QALYs ( $\mathrm{x}$ axis) and the difference in costs (y-axis) between T2T and UC. Figure 2A shows that after $94 \%$ of the two years' bootstrapped ratios were situated in the upper-right quadrant. Figure 2B shows that $66 \%$ of the three years' bootstrapped ratios were situated in the lower-right quadrant, which signifies lower costs and higher effectiveness.

\section{Sub-analysis}

A sub-analysis was performed on patients that were included in the cohorts from 2006. Table 1 presents the baseline characteristics of the $69 \mathrm{UC}$ patients that were included in the UC cohort from 2006. The 


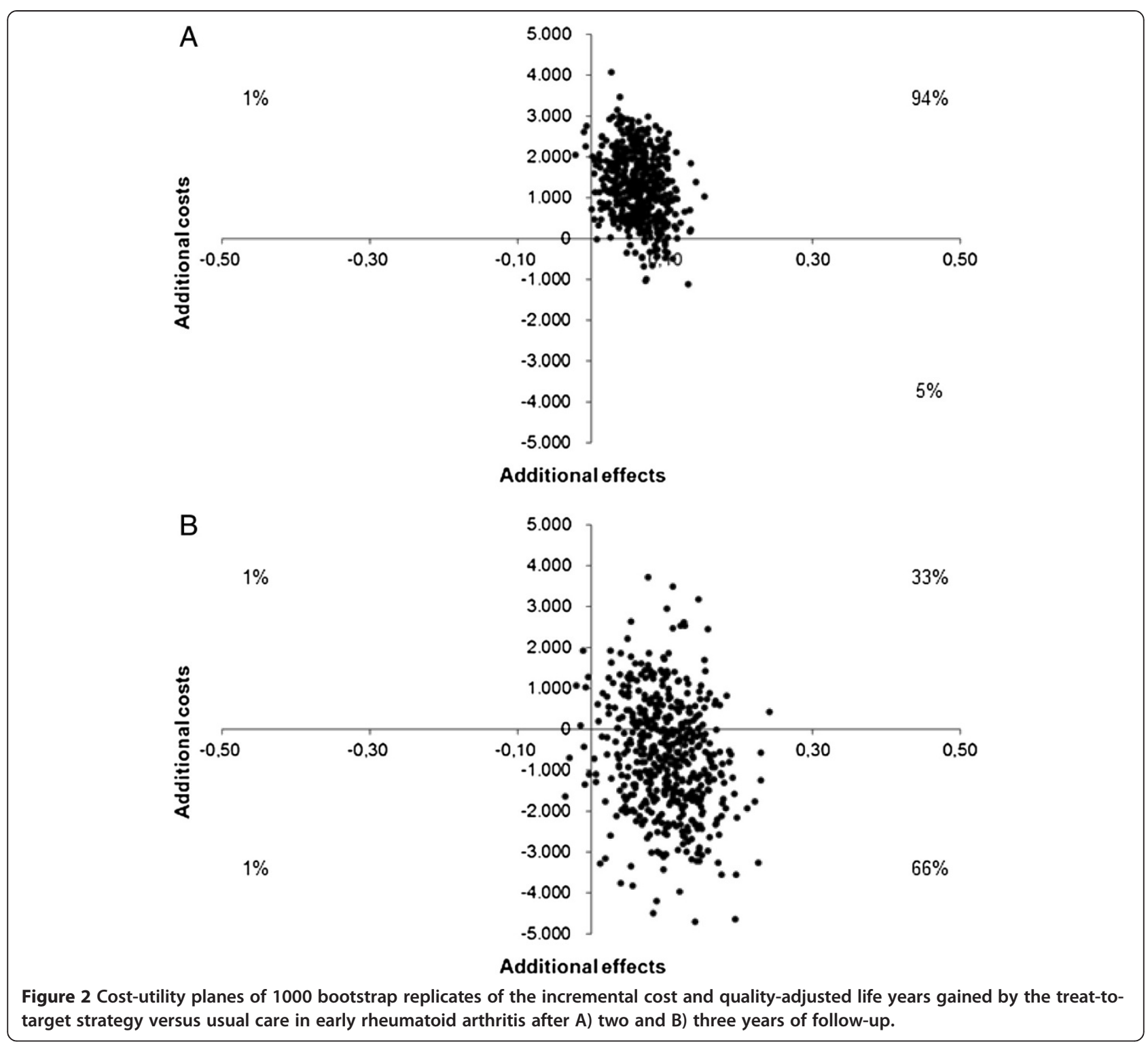

health outcomes of these patients are presented in Table 2 and the amount of care consumption and mean costs per patient during two and three years of follow-up are presented in Table 3. After two years of follow-up, the ICER was $€ 8,709$ per patient in remission. After three years T2T was dominant (this analysis included 45 UC patients). We were not able to perform analysis on the ICURs because of the low number of patients with data with on QALYs in the UC group.

\section{Discussion}

Our study suggests that treating to the target of remission is the preferred strategy over usual care in early RA. After two years of treatment, T2T is cost-effective as it comes with higher costs but also with substantially higher effectiveness. In the T2T group DAS28 remission had been achieved more frequently and there was a larger gain in health-related QALYs compared with UC. The ICUR lies far below the threshold of $€ 80,000$ per QALY, which is considered to be an acceptable willingness to pay for one QALY in The Netherlands [25]. Moreover, the costs to bring one more patient in remission also seem to be acceptable. Results of an extended follow-up analysis of three year data were clearly in favor of $\mathrm{T} 2 \mathrm{~T}$, with $64 \%$ chance of the T2T strategy coming at lower costs with higher effectiveness compared to UC. To our knowledge, this is the first health economic evaluation of comparing T2T with UC using real-life data.

The drivers of absolute costs and cost differences between T2T and UC were anti-TNF therapy and hospitalization. Our previous studies demonstrated that 
the majority of the T2T patients achieved remission with conventional DMARDs [7,9]. According to the treatment protocol, anti-TNF was prescribed only for a minority of patients whose disease activity remained moderate to high after insufficient effect of conventional DMARDs, thereby preventing overtreatment with antiTNF agents with their costs and side effects. In the UC group, anti-TNF was initiated later in the disease course, and, therefore, it might be less effective and longer required in patients. Costs due to hospitalization were directly related to RA. The higher number of hospital admissions in the UC group might be explained by less efficient disease control. Data on intra-articular injections were not available. However, we do not think this can explain the difference in costs between T2T and UC, because of the low costs of injections.

The principle of T2T is to aim at achieving and sustaining remission as early as possible. Our expectation is that the extra effort and time spent in the first years of the disease, ultimately result in a reduction of the number of consultations later in the disease course and the possibility of tapering and discontinuing medication in case of sustained remission, thereby diminishing costs. Therefore, we expect that on the long-term, cost savings associated with T2T will increase. Furthermore, better and earlier disease control might lead to more work participation on the long-term, which will ultimately lower the costs of T2T for society and improve quality of life for the patients.

An important strength of this study is the quasiexperimental design containing real-life observational data regarding effectiveness, health-related quality of life and costs of T2T compared with usual care. Moreover, in the DREAM registry all consecutive patients are prospectively followed and a standardized data set is collected. This is in contrast to many health economic evaluations, which often use modeling techniques with many underlying assumptions or use clinical trial data of highly selected patients.

However, this study has some limitations also. Obviously, since our patients are unselected, randomisation between the comparing cohorts was not performed with the risk of confounding by indication present in the comparison. However, participation in either one of the cohorts was determined by living area while patients attended comparable rheumatology clinics, all participating in the DREAM registry and working within the same health care system. We assume that this has limited the possibility of confounding by indication. Furthermore, no relevant differences in baseline characteristics which are prognostic for the treatment effect were found. Second, it should be noted that UC patients were included from 2000 until present, whereas T2T patients were included from 2006 until present. Even though the same treatment options for both groups were available during observation and anti-TNF guidelines have not been changed since 2000, one can assume that UC has changed between 2000 and 2006. A sub-analysis omitting UC patients recruited prior to 2006 showed comparable ICERs, however with less statistical power, leading to the same conclusions. Therefore, this study provides the best possible comparison currently available. A third limitation is that a preference based health-related quality of life measure was not available in the UC cohort, and, therefore, we estimated utilities from the HAQ. The HAQ has been shown to be highly correlated with health state utility values, which are used to calculate QALYs [26]. Nevertheless, HAQ-derived utilities will only capture change in quality of life generated by the patient's functional status and not by other factors. We expect that T2T patients improve at more dimensions of quality of life than only function status. Therefore, we believe that this was a conservative analysis. We acknowledge that the use of a mapping method will always be suboptimal to primary collection of utility data. Fourth, we applied a health care perspective, thereby taking into account only direct medical costs. However, the economic burden of RA goes beyond health care costs [27-29] and a societal perspective would be preferable. RA leads to substantial losses in terms of work productivity which increases with disease duration [30,31]. Unfortunately, data on work participation were not available. According to our view, we performed a conservative cost analysis and our expectations are that T2T, which decreases disease activity rapidly and early in the disease course, will have an additional positive effect on non-medical costs (e.g. work productivity, informal care, and paid housekeeping).

\section{Conclusions}

We performed a health economic study of T2T versus UC, using observational data of the DREAM registry. We conclude that treating to the target of remission in early RA is cost-effective as compared with usual care at the discretion of the attending physician.

\section{Appendix}

\section{Cost prices 2011}

Consult at rheumatologist (13 minutes) $€ 66.90$

Consult at rheumatology nurse (20 minutes) $€ 66.90$

Telephonic consult at rheumatologist (5 minutes) $€ 25.73$

Hospital day care related to biologics $€ 122.13$

Hospital admission (one day) $€ 454.69$

\section{Competing interests}

An unrestricted educational grant was provided by Abbott, The Netherlands, which was used to support MV.

The authors declare that they have no competing interests.

\section{Authors' contributions}

MV contributed to the design of the study, data collection, analysis and interpretation, and drafted the manuscript. WK contributed to the design of the study, data collection, analysis and interpretation, and drafted the 
manuscript. HHK contributed to the design of the study, data collection and interpretation, and drafted the manuscript. LMAB-J contributed to the design of the study, data interpretation, and critically revised the manuscript. HJBM contributed to data collection and interpretation, and critically revised the manuscript. TRZ contributed to data collection and interpretation, and critically revised the manuscript. AAdB contributed to data collection and interpretation, and critically revised the manuscript. PLCMvR contributed to the design of the study, data collection and interpretation, and critically revised the manuscript. JF contributed to the design of the study, data interpretation, and critically revised the manuscript. MAFJvdL contributed to the design of the study, data collection and interpretation, and drafted the manuscript. All authors read and approved the final manuscript.

\section{Acknowledgements}

We would like to thank all patients, rheumatology nurses, and rheumatologists who participated in this study. We are grateful to Eddy Adang, health economist at the department of Health Evidence, Radboud University Nijmegen Medical Centre, The Netherlands, for providing the bootstrap Macro in Excel and advising us in using it.

\section{Author details}

${ }^{1}$ Arthritis Center Twente, Department of Psychology, Health and Technology, University of Twente, Enschede, The Netherlands. ${ }^{2}$ Department of Rheumatology and Clinical Immunology, Medisch Spectrum Twente, Enschede, The Netherlands. ${ }^{3}$ Department of Rheumatology, Radboud University Nijmegen Medical Centre, Nijmegen, The Netherlands. ${ }^{4}$ Department of Rheumatology, Ziekenhuisgroep Twente, Hengelo, The Netherlands. ${ }^{5}$ Department of Rheumatology, Isala Klinieken, Zwolle, The Netherlands. ${ }^{6}$ Department of Rheumatology, Maartenskliniek, Nijmegen, The Netherlands.

Received: 18 February 2013 Accepted: 3 December 2013 Published: 13 December 2013

\section{References}

1. Smolen JS, Aletaha D, Bijlsma JW, et al: Treating rheumatoid arthritis to target recommendations of an international task force. Ann Rheum Dis 2010, 69:631-637.

2. Schoels M, Knevel R, Aletaha D, et al: Evidence for treating rheumatoid arthritis to target: results of a systematic literature search. Ann Rheum Dis 2010, 69:638-643.

3. Knevel R, Schoels M, Huizinga TW, et al: Current evidence for a strategic approach to the management of rheumatoid arthritis with diseasemodifying antirheumatic drugs: a systematic literature review informing the EULAR recommendations for the management of rheumatoid arthritis. Ann Rheum Dis 2010, 69:987-994.

4. Schipper LG, van Hulst LT, Grol R, et al: Meta-analysis of tight control strategies in rheumatoid arthritis: protocolized treatment has additional value with respect to the clinical outcome. Rheumatology (Oxford) 2010, 49:2154-2164.

5. Goekoop-Ruiterman YP, de Vries-Bouwstra JK, Kerstens PJ, et al: DAS-driven therapy versus routine care in patients with recent-onset active rheumatoid arthritis. Ann Rheum Dis 2010, 69:65-69.

6. Soubrier M, Lukas C, Sibilia J, et al: Disease activity score-driven therapy versus routine care in patients with recent-onset active rheumatoid arthritis: data from the GUEPARD trial and ESPOIR cohort. Ann Rheum Dis 2011, 70:611-615.

7. Schipper $L$, Vermeer $M$, Kuper $H$, et al: A tight control treatment strategy aiming for remission in early rheumatoid arthritis is more effective than usual care treatment in daily clinical practice: a study of two cohorts in the Dutch Rheumatoid Arthritis Monitoring (DREAM) registry. Ann Rheum Dis 2011, 71:845-850.

8. Prevoo ML, van 't Hof MA, Kuper $\mathrm{HH}$, et al: Modified disease activity scores that include twenty-eight-joint counts. Development and validation in a prospective longitudinal study of patients with rheumatoid arthritis. Arthritis Rheum 1995, 38:44-48.

9. Vermeer $\mathrm{M}$, Kuper $\mathrm{HH}$, Hoekstra $\mathrm{M}$, et al: Implementation of a treat-totarget strategy in very early rheumatoid arthritis: results of the Dutch Rheumatoid Arthritis Monitoring remission induction cohort study. Arthritis Rheum 2011, 63:2865-2872.
10. van Tuyl LH, Boers M, Lems WF, et al: Survival, comorbidities and joint damage 11 years after the COBRA combination therapy trial in early rheumatoid arthritis. Ann Rheum Dis 2010, 69:807-812.

11. Krishnan $E$, Lingala $B$, Bruce $B$, Fries JF: Disability in rheumatoid arthritis in the era of biological treatments. Ann Rheum Dis 2012, 71:213-218.

12. Strand $V$, Khanna D: The impact of rheumatoid arthritis and treatment on patients' lives. Clin Exp Rheumatol 2010, 28:S32-S40.

13. Korthals-de Bos I, Van Tulder M, Boers M, et al: Indirect and total costs of early rheumatoid arthritis: a randomized comparison of combined stepdown prednisolone, methotrexate, and sulfasalazine with sulfasalazine alone. J Rheumatol 2004, 31:1709-1716.

14. Welsing PM, van Riel PL: The Nijmegen inception cohort of early rheumatoid arthritis. J Rheumatol Supp/ 2004, 69:14-21.

15. Arnett FC, Edworthy SM, Bloch DA, et al: The American Rheumatism Association 1987 revised criteria for the classification of rheumatoid arthritis. Arthritis Rheum 1988, 31:315-324.

16. Fransen J, Creemers MC, Van Riel PL: Remission in rheumatoid arthritis: agreement of the disease activity score (DAS28) with the ARA preliminary remission criteria. Rheumatology (Oxford) 2004, 43:1252-1255.

17. Cartwright WS: Methods for the economic evaluation of health care programmes, second edition. In Methods for the economic evaluation of health care programmes, second edition. Edited by Michael F, Drummond, Bernie O'B, Greg L, Stoddart, George W. Torrance. Oxford: Oxford University Press; 1997. J Ment Health Policy Econ 1999; 2: 43.

18. Brooks R: EuroQol: the current state of play. Health Policy 1996, 37:53-72.

19. Fries JF, Spitz P, Kraines RG, Holman HR: Measurement of patient outcome in arthritis. Arthritis Rheum 1980, 23:137-145.

20. Siegert CE, Vleming LJ, Vandenbroucke JP, Cats A: Measurement of disability in Dutch rheumatoid arthritis patients. Clin Rheumatol 1984, 3:305-309.

21. Bansback N, Marra C, Tsuchiya A, et al: Using the health assessment questionnaire to estimate preference-based single indices in patients with rheumatoid arthritis. Arthritis Rheum 2007, 57:963-971.

22. Harrison MJ, Lunt M, Verstappen SM, et al: Exploring the validity of estimating EQ-5D and SF-6D utility values from the health assessment questionnaire in patients with inflammatory arthritis. Health Qual Life Outcomes 2010, 8:21.

23. Dutch Board of Health Insurances: Dutch Guideline for Cost Analyses; 2010.

24. Statistics Netherlands: Statistics Netherlands. www.cbs.nl (accessed 28 November 2011

25. Raad voor Volksgezondheid en Zorg: Zinnige en Duurzame Zorg; 2006.

26. Marra CA, Woolcott JC, Kopec JA, et al: A comparison of generic, indirect utility measures (the HUI2, HUI3, SF-6D, and the EQ-5D) and diseasespecific instruments (the RAQoL and the HAQ) in rheumatoid arthritis. Soc Sci Med 2005, 60:1571-1582.

27. Kvien TK: Epidemiology and burden of illness of rheumatoid arthritis. Pharmacoeconomics 2004, 22:1-12.

28. Zhang W, Anis AH: The economic burden of rheumatoid arthritis: beyond health care costs. Clin Rheumatol 2011, 30(Suppl 1):S25-32.

29. Boonen A, Severens $J$ : The burden of illness of rheumatoid arthritis. Clin Rheumatol 2011, 30(Suppl 1):S3-8.

30. Verstappen SM, Bijlsma JW, Verkleij H, et al: Overview of work disability in rheumatoid arthritis patients as observed in cross-sectional and longitudinal surveys. Arthritis Rheum 2004, 51:488-497.

31. Neovius M, Simard JF, Askling J: How large are the productivity losses in contemporary patients with RA, and how soon in relation to diagnosis do they develop? Ann Rheum Dis 2011, 70:1010-1015.

doi:10.1186/1471-2474-14-350

Cite this article as: Vermeer et al.: Treating to the target of remission in early rheumatoid arthritis is cost-effective: results of the DREAM registry. BMC Musculoskeletal Disorders 2013 14:350. 